\title{
Calculation of the Grounding Resistance of Structures Located on Elevated Terrain
}

\author{
Antonio Sunjerga, Farhad Rachidi, Fellow, IEEE, Marcos Rubinstein, Fellow, IEEE and Dragan Poljak, \\ Senior Member, IEEE
}

\begin{abstract}
We present an analysis of the response of a grounding electrode located on top of a mountain. Specifically, we derive an analytical solution for the low-frequency response of a hemispheric grounding electrode buried on the top of a coneshaped mountain characterized by its apex angle. The derived equation is validated using numerical simulations based on the Finite Element Method obtained using COMSOL.

Simulation results show that, for the same ground electrical parameters, the grounding resistance of such an electrode for steep mountains can be significantly higher than that obtained if the electrode is on flat ground. Such situations can occur in particular for the case of telecommunication towers or wind turbines located on mountaintops.

The study emphasizes the importance of considering the terrain profile in the evaluation of the grounding resistance of structures located in elevated locations.
\end{abstract}

Index Terms-Lightning, grounding resistance, hilly, elevated terrain, grounding electrode

\section{INTRODUCTION}

$\mathrm{T}$ all structures such as wind turbines and mobile phone base stations are often installed in remote and hilly locations. Those locations are very likely to be struck by lightning due to their geographical elevation $[1,2]$ and to the initiation of upward flashes $[3,4]$ from them.

These hilly areas are often very rocky with low soil conductivity $(0.001 \mathrm{~S} / \mathrm{m}$ and lower). Therefore, the design of proper grounding systems is of high importance.

The evaluation of the impedance of grounding electrodes requires, in general, the application of numerical methods (e.g., [5]), or simplified transmission-line-based or circuit-based models (e.g., [6]). On the other hand, analytical expressions are available for the grounding resistance for various electrode geometries, such as a hemisphere, buried ring, and vertical or horizontal rods (see, e.g., Appendix D of [7]). All these analytical expressions have been derived assuming that the earth is a homogeneous half space and its surface is flat.

To the best of our knowledge, no previous studies have discussed the influence of a non-flat ground on the impedance of grounding electrodes. In this paper, we derive an analytical solution for the case of a hemispheric electrode located on top of a mountain represented by a conical shape. We compare the derived analytical solution to numerical simulations for validation purposes.

\section{LOW-FREQUENCY RESPONSE OF A GROUNDING ELECTRODE}

\section{A. Basic Equations}

The low-frequency response of a grounding system is governed by Ohm's law and the current continuity equation. Ohm's law in differential form, also called point form, can be written as:

$$
\vec{J}=\sigma \vec{E}
$$

where $\sigma$ is the conductivity of the medium at a given point and $\vec{J}$ is the current density.

The continuity equation in cases where the time derivative of the volume charge density can be neglected, can be written as:

$$
\vec{\nabla} \cdot \vec{J}=0
$$

Using (1) and expressing the electric field in terms of the electric potential for the considered low frequency regime, (2) can be rewritten as

$$
-\vec{\nabla} \cdot(\sigma \vec{\nabla} \cdot \varphi)=0
$$

Current sources at the boundary with a non-conducting medium can be imposed through Neumann boundary condition. In the analysis, the numerical simulations will be carried out using the $\mathrm{AC} / \mathrm{DC}$ module of the commercial tool COMSOL [8].

\section{B. Hemispheric Grounding Electrode in a Flat Ground}

The geometry of the problem is shown in Figure 1. We consider a metallic hemispheric electrode of radius $R_{0}$ buried in a flat ground, characterized by its electric conductivity and relative permittivity. The derivation of the grounding resistance is classical and has been carried out elsewhere $[9,10]$. However, for the sake of completeness, we will present it here.

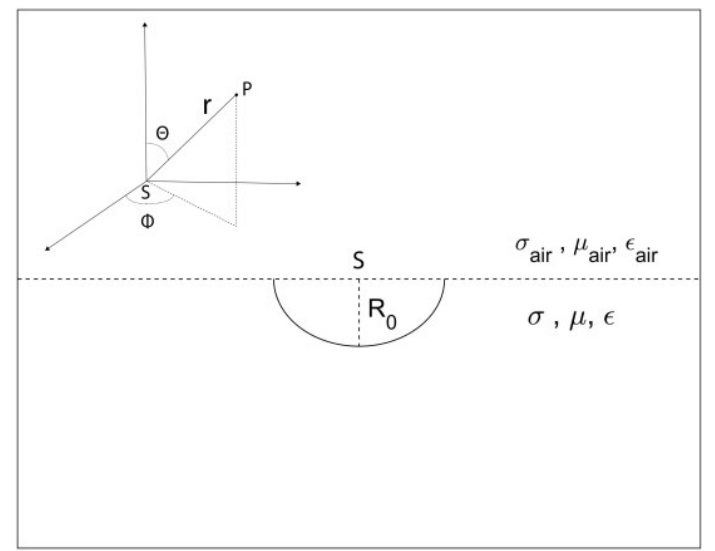

Fig. 1. Hemispheric electrode buried in a flat ground. The origin of the spherical coordinate system is at the center of the hemisphere. 
A current $I$ is impressed at the center of the hemisphere. The resistance of the grounding electrode is defined as the ratio of the ground potential rise (GPR) at the feeding point and the injected current $I$ :

$$
R=\frac{V_{\infty}}{I}
$$

Due to the symmetry of the problem, the magnitude of the current density $\vec{J}$ is constant at points in the ground that are at a given distance $r$ from the origin and it is collinear with the radial vector. Therefore, the current at a distance $r$ is simply given by:

$$
\vec{J}=\frac{I}{A} \widehat{e_{r}}=\frac{I}{2 \pi r^{2}} \widehat{e_{r}}
$$

where $A$ is the surface of the hemisphere at a given distance $r$. Now, from Ohm's law (1), one obtains:

$$
\vec{E}=\frac{I}{2 \pi \sigma r^{2}} \widehat{e_{r}}
$$

The GPR is defined as the voltage difference from the feeding point to the remote earth:

$$
\begin{gathered}
V_{\infty}=\int_{r=0}^{r=\infty} \vec{E} \overrightarrow{d r}= \\
=\int_{r=0}^{r=R_{0}} \frac{I}{2 \pi \sigma_{\text {hemisphere }} r^{2}} d r+\int_{r=R_{0}}^{r=\infty} \frac{I}{2 \pi \sigma r^{2}} d r
\end{gathered}
$$

The hemisphere being made of metal $\left(\sigma_{\text {hemisphere }}\right.$ in the order of $10^{6} \mathrm{~S} / \mathrm{m}$ ), the first integral on the right-hand side of the equation can be neglected (the voltage is constant along the conductor). The final result reads:

$$
V_{\infty} \approx \frac{I}{2 \pi \sigma R_{0}}
$$

Thus, the resistance is given by:

$$
R_{\text {flat }}=\frac{V_{\infty}}{I}=\frac{1}{2 \pi \sigma R_{0}}
$$

\section{Hemispheric Grounding Electrode in a Cone-Shaped Ground}

Let us now consider the geometry shown in Figure 2. In this case, a hemispheric grounding electrode of radius $R_{0}$ is buried on the top of a truncated cone-shaped earth characterized by an angle $\varphi$. We assume that the hemispheric electrode reaches the edges of the truncated cone, a situation which does not necessarily correspond to a realistic case, but will allow us to derive an analytical solution for the grounding resistance, providing insight into the effect of a non-flat terrain on the lowfrequency response of a grounding system. A more realistic situation will be considered in Section II.E and analyzed numerically using COMSOL.

The current is assumed to be applied at the center of the hemisphere. In this case, the current density in the spherical coordinate system will be a function of both $r$ and the azimuth angle.

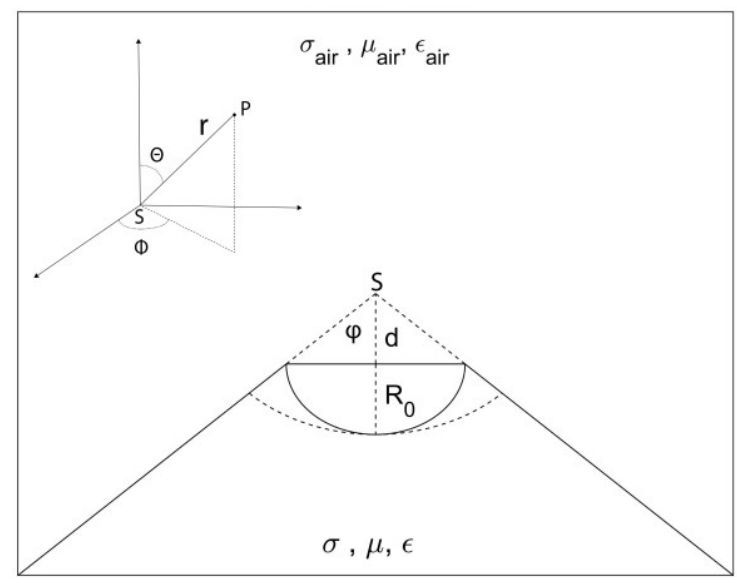

Fig. 2. Hemispheric electrode buried on the top of a truncated cone-shaped ground. The center of the spherical coordinate system is at the tip of the untruncated cone.

In order to be able to derive an analytical solution for the grounding resistance, let us approximate the original hemispheric electrode by a spherical sector delimited by the dotted line and centered at the tip of the cone (Fig. 2). The radius of this sphere is given by

$$
R_{1}=R_{0}+d
$$

in which $\mathrm{d}$ is defined in Fig. 2. $R_{1}$ can be expressed in terms of the apex angle $\varphi$ as follows:

$$
R_{1}=R_{0}(1+\operatorname{ctg}(\varphi))
$$

As can be seen from Fig. 2, the approximated electrode covers a larger area compared to the original one. Thus, the resulting grounding resistance would be an underestimate of the original one. A curved surface area of a spherical sector of radius $r$ is given by:

$$
A=\Omega r^{2}
$$

where $\Omega$ is the solid angle which can be calculated from the apex angle as:

$$
\Omega=2 \pi(1-\cos (\varphi))
$$

Because of the symmetry of the approximate geometry, the current density depends only on the variable $r$ and it is always collinear with the radial vector. The current density at a given distance $r$ is given by

$$
\vec{J}=\frac{I}{A} \widehat{e_{r}}=\frac{I}{2 \pi(1-\cos (\varphi)) r^{2}} \widehat{e_{r}}
$$

The GPR at the feeding point can be calculated as:

$$
V_{\infty}=\int_{r=0}^{r=\infty} \vec{E} \overrightarrow{d r} \approx \int_{r=R_{1}}^{r=\infty} \frac{I}{2 \pi \sigma(1-\cos (\varphi)) r^{2}} d r
$$

where, as in (7), the potential drop across the conductor is considered to be negligible. The final expression for the voltage is given by

$$
V_{\infty}=\frac{I}{2 \pi \sigma R_{0}(1-\cos (\varphi))(1+\operatorname{ctg}(\varphi))}
$$


which can be used in (4) to calculate the resistance of the grounding electrode as follows:

$$
R_{\text {cone }}=\frac{1}{2 \pi \sigma R_{0}(1-\cos (\varphi))(1+\operatorname{ctg}(\varphi))}
$$

To express the increase of the grounding resistance as a function of the ground profile, let us define a coefficient $k$ given by the ratio of the resistance for a flat ground and the one for a conical ground:

$$
k=\frac{R_{\text {cone }}}{R_{\text {flat }}}=\frac{1}{(1-\cos (\varphi))(1+\operatorname{ctg}(\varphi))}
$$

As can be seen from (18), this ratio depends only on the apex angle $\varphi$. For the case of a flat ground, $\varphi=90^{\circ}$, (17) reduces to (9) and $k$ tends to 1 . Fig. 3 presents the coefficient $\mathrm{k}$ as a function of the apex angle.

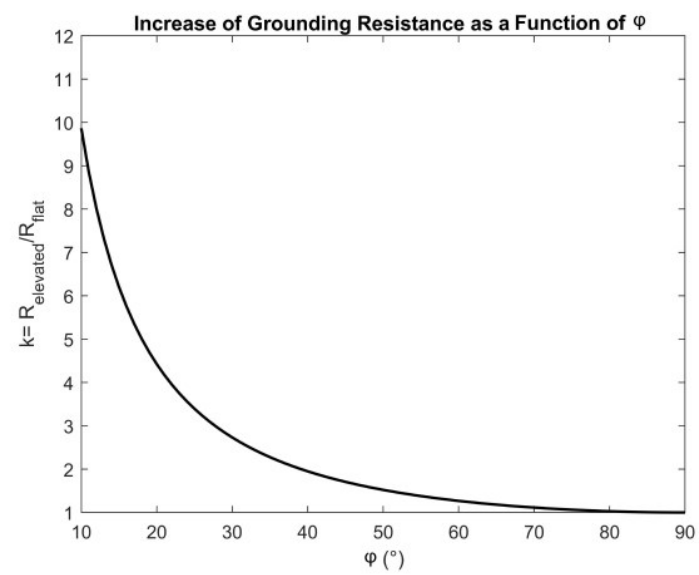

Fig. 3. Coefficient of increase of the grounding resistance as a function of the apex angle.

It can be seen that for very steep profiles, the ratio can take significant values, corresponding to an appreciable increase in the grounding resistance. As an example, for $\varphi=60^{\circ}$, the resistance is increased by about $30 \%$, and for $\varphi=40^{\circ}$, the increase is almost $100 \%$. For very steep profiles, namely for angles smaller than $20^{\circ}$, the resistance can be increased by a factor of 5 or more.

\section{Comparison with Numerical Simulations}

Fig. 4 shows the distribution of the potential to remote earth obtained by solving (3) numerically using COMSOL. A current source of 1 A was applied at the center of the $20-\mathrm{m}$ radius hemispheric electrode. The Dirichlet boundary condition is set at the bottom of the cone as $\varphi=0 \mathrm{~V}$, and the Neumann boundary condition, $\vec{J}_{\text {normal }}=0$, is set on the surface of the cone. The height of the cone is taken such that it is far enough for the results to converge (200 to $2000 \mathrm{~m}$, depending on the apex angle). Let us consider a hemisphere with a radius $\mathrm{R}_{0}=20 \mathrm{~m}$, which would represent as a first approximation the grounding system of a wind turbine. Considering a grounding conductivity of $\sigma=0.001 \mathrm{~S} / \mathrm{m}$ and an apex angle of $\varphi=45^{\circ}$, we obtain a grounding resistance of $13,68 \Omega$, while using the analytical approximation (18), we obtain a value of $13,58 \Omega$.

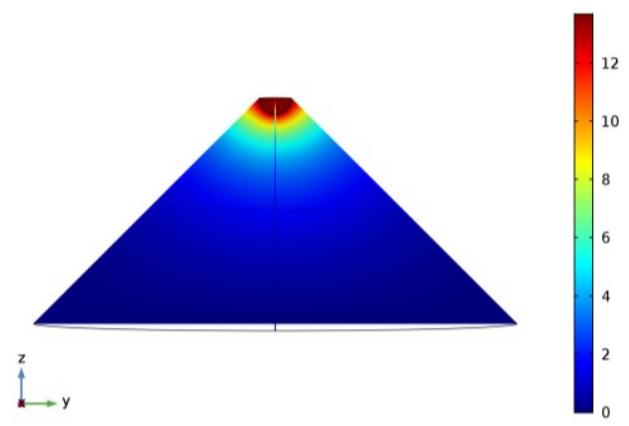

Fig. 4. Potential to remote earth for the case of $R_{0}=20 \mathrm{~m}, \sigma=0.001 \mathrm{~S} / \mathrm{m}, \varphi=45^{\circ}$ and $1 \mathrm{~A}$ current source applied at the center of the hemisphere. Simulated in the commercial tool COMSOL [8].

Fig 5 shows the values of the grounding resistance as a function of the apex angle, obtained using the proposed analytical solution and the COMSOL numerical results. It can be seen that the proposed analytical approximation yields values which are in excellent agreement with numerical results, for all considered angles.

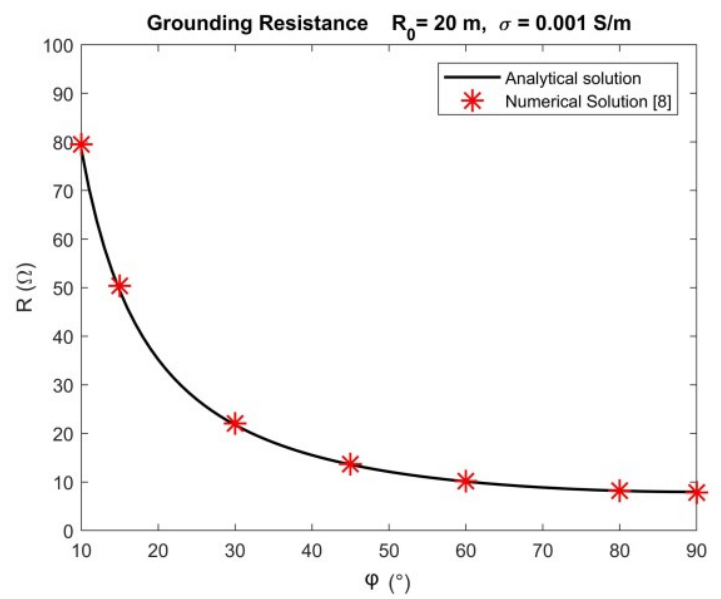

Fig. 5. Grounding resistance for $\sigma=0.001 \mathrm{~S} / \mathrm{m}$ and $\mathrm{R}_{0}=20 \mathrm{~m}$. Analytical solution and discrete numerical solution as a function of apex angle.

Fig 6 shows similar results for the case of a hemispheric grounding electrode of radius $R_{0}=5 \mathrm{~m}$. Again, it can be seen that the results obtained using the proposed analytical expression agree well with the numerical results obtained using COMSOL.

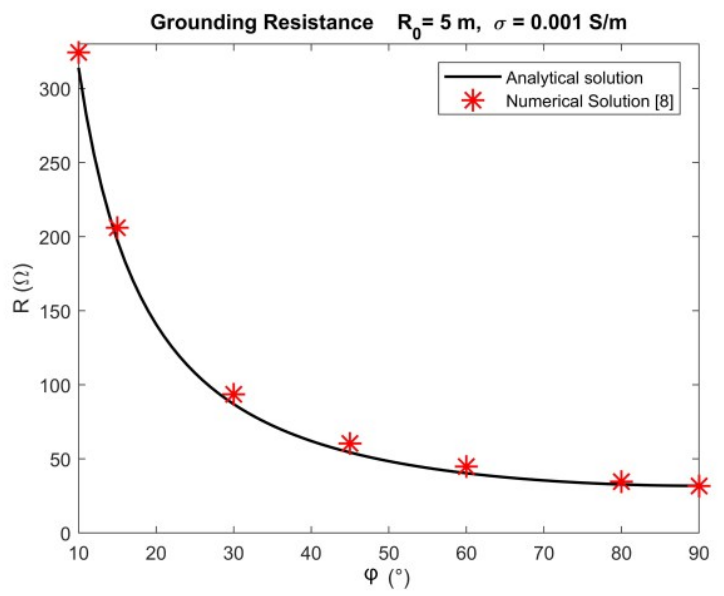

Fig. 6. Grounding resistance for $\sigma=0.001 \mathrm{~S} / \mathrm{m}$ and $\mathrm{R}_{0}=5 \mathrm{~m}$. Analytical solution and discrete numerical solution as a function of the apex angle. 


\section{E. Variation of Cone Top Radius}

As mentioned earlier, the considered case of a hemispheric electrode that reaches the edges of the truncated cone-shaped mountain (as opposed to one that is smaller) is not a realistic case. Here, we will consider the geometry shown in Fig. 7 in which the top radius of the cone is bigger than the radius of hemispheric grounding electrode. The distance between the center of the hemisphere and edge of cone is $r_{\text {top }}$ and the apex angle is $\varphi$.

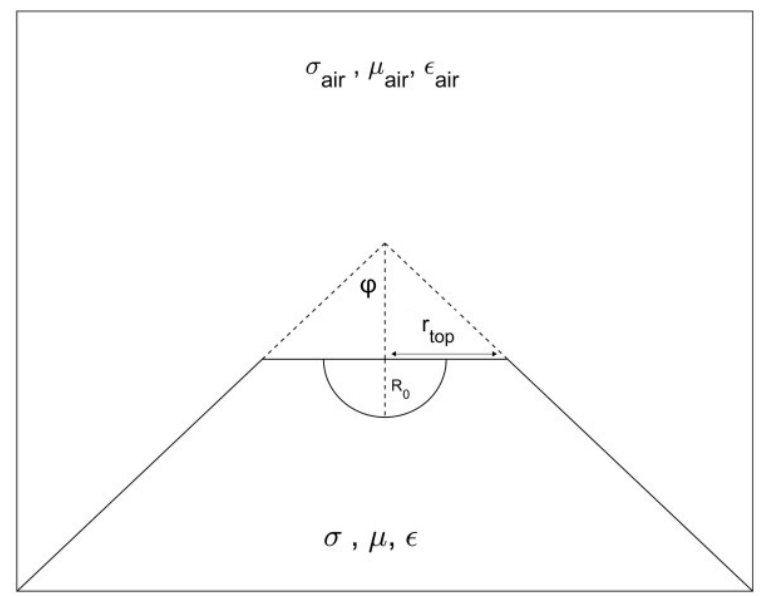

Fig. 7. Hemispheric electrode buried on the top of a truncated cone-shaped ground. The center of the spherical coordinate system is at the tip of the truncated cone prolongation.

Table 1 shows the COMSOL computed values for the grounding resistance of a 5-m radius hemispheric grounding electrode, with a ground conductivity of $\sigma=0.001 \mathrm{~S} / \mathrm{m}$, as a function of the apex angle and the distance $r_{\text {top }}$ to the cone edge. The grounding resistance of the same electrode buried in a flat ground would be $31.8 \Omega$.

Table 1 - Grounding resistance of a 5-m radius hemisphere grounding electrode buried in a cone-shaped ground of conductivity $\sigma=0.001 \mathrm{~S} / \mathrm{m}$, as a function of the apex angle $\varphi$ and the distance $r_{\text {top }}$ to the cone edge.

\begin{tabular}{lcccc}
\hline \multirow{2}{*}{$\begin{array}{l}\text { Apex } \\
\text { angle }\end{array}$} & \multicolumn{4}{c}{ Resistance $(\Omega)$} \\
\cline { 2 - 5 }$(\varphi)$ & $r_{\text {top }}=\mathrm{R}_{0}$ & $r_{\text {top }}=2 \mathrm{R}_{0}$ & $r_{\text {top }}=4 \mathrm{R}_{0}$ & $r_{\text {top }}=10 \mathrm{R}_{0}$ \\
\hline $60^{\circ}$ & 44.8 & 38 & 34.5 & 32.4 \\
$45^{\circ}$ & 60.4 & 46.2 & 38.6 & 34.1 \\
$30^{\circ}$ & 93.5 & 62.9 & 46.6 & 36.8 \\
$15^{\circ}$ & 206 & 121 & 75.7 & 48.7 \\
$10^{\circ}$ & 324.4 & 188.7 & 108.7 & 67.9 \\
\hline
\end{tabular}

It can be seen that, even for the case when the top radius is four times the radius of the grounding electrode, the increase in the grounding resistance with respect to the case of a flat ground would be as high as $50 \%$ for an apex angle of $30^{\circ}$. For the case of an apex angle of $60^{\circ}$ and $r_{\text {top }}=10 R_{0}$, the resulting grounding resistance approaches the value for a flat ground. Similar relative increase as in Table 1. was observed for the case of vertical rod and it will be further discussed in future studies.

\section{CONCLUSION}

In this study, we analyzed the response of a grounding electrode located on top of a hill or a mountain top. Specifically, we derived an analytical solution for the low-frequency response of a hemispheric grounding electrode buried on top of a cone-shaped mountain characterized by its apex angle. The derived equation was validated using numerical simulations based on the Finite Element Method obtained using COMSOL.

Results show that, for the same ground electrical parameters, the grounding resistance of such an electrode can increase significantly for steep mountains. Such situations can occur in particular for the case of telecommunication towers or wind turbines located on mountaintops. These conclusions can be extended to any geometry of grounding system.

The study emphasizes the importance of considering the terrain profile in the evaluation of the grounding resistance or structures located in elevated locations.

Acknowledgments - This work was supported in part by the Swiss National Science Foundation (Project No. 200020_175594).

\section{REFERENCES}

[1] R. M. Reap, "Evaluation of Cloud-to-Ground Lightning Data from the Western United States for the 1983-84 Summer Seasons," Journal of Climate and Applied Meteorology, vol. 25, pp. 785-799, Jun. 1986.

[2] A. Smorgonskiy, F. Rachidi, M. Rubinstein, and G. Diendorfer, "On the relation between lightning flash density and terrain elevation," in 2013 International Symposium on Lightning Protection (XII SIPDA), 2013.

[3] F. Rachidi, M. Rubinstein and A. Smorgonskiy, Lightning Protection of Large Wind-Turbine Blades, in Wind Energy Conversion Systems, Springer, p. 227-242, 2012.

[4] J. Montanyà, O. van der Velde, and E. R. Williams, "Lightning discharges produced by wind turbines," Journal of Geophysical Research: Atmospheres, vol. 119, no. 3, pp. 1455-1462, Feb. 2014.

[5] L. Grcev and V. Arnautovski-Toseva, "Grounding systems modeling for high frequencies and transients: some fundamental considerations," in 2003 IEEE Bologna Power Tech Conference Proceedings

[6] L. D. Grcev, A. Kuhar, V. Arnautovski-Toseva, and B. Markovski, "Evaluation of High-Frequency Circuit Models for Horizontal and Vertical Grounding Electrodes," IEEE Transactions on Power Delivery, pp. 1-1, 2018.

[7] F. M. Tesche, M. Ianoz, T. Karlsson EMC Analysis Methods and Computational Models, N.J. Wiley, 1997.

[8] COMSOL Multiphysics ${ }^{\circledR}$ v.5.3. www.comsol.com. COMSOL AB, Stockholm, Sweden.

[9] E. D. Sunde, Earth Conduction Effects in Transmission Systems. New York: Van Nostrand, 1949.

[10] D. Poljak Advanced modeling in computational electromagnetic compatibility, N.J. Wiley, 2007. 